\title{
Refletir a Prática Clínica: a Inovação na Intervenção
}

\author{
Ver artigo relacionado \\ na página 256
}

\author{
Gustavo B. F. Oliveira', Álvaro Avezum
}

0 artigo "Construção e Validação de um Sistema Integrado de Dados de Intervenção Coronária Percutânea no Brasil (Registro ICP-BR): Perfil Clínico dos Primeiros 1.249 Pacientes Incluídos", de autoria de Paula et al. ${ }^{1}$, publicado nesta edição da Revista Brasileira de Cardiologia Invasiva, conduzido por meio de esforço colaborativo e eficiente de oito instituições brasileiras, permite algumas reflexões relacionadas à prática clínica da intervenção coronária percutânea.

Dentro das estratégias de pesquisa epidemiológica em medicina cardiovascular, os estudos randomizados adequadamente conduzidos e com poder estatístico suficiente permitem a avaliação da eficácia e da segurança de intervenções médicas. Apesar do avanço em pesquisa proporcionado por tais estudos, uma das limitações potenciais dessa estratégia pode residir na falta de generalização externa dos resultados, também descrita como aplicabilidade para a prática clínica. Alguns estudos randomizados permitem aplicabilidade substancial pela característica pragmática, entretanto esse não é um achado comum em medicina cardiovascular.

Consequentemente, avaliações que possam mensurar a prática clínica, dentro do denominado "mundo real" da intervenção coronária percutânea, se tornam necessárias e cruciais para que, reconhecendo o que acontece em ambiente menos controlado e menos enviesado, possamos planejar e implementar estratégias de melhoria da prática clínica, que permitam cobrir a lacuna existente entre conhecimento científico e benefícios estendidos à população.

O manuscrito de Paula et al.' oferece oportunidade única para preencher tal lacuna. Apesar da disponibilidade de estudos transversais (registros) na área em questão, como o Registro CENIC (Central Nacional de Intervenções Cardiovasculares), a característica espontânea e voluntária de envio dos dados desses estudos não permite reflexo adequado da prática clínica. Portanto, estudos transversais que permitam a inclusão de todos os pacientes submetidos a intervenção coronária percutânea são desejáveis para corretamente mensurar o "mundo real" desse procedimento na população com doença arterial coronária estável ou instável.
Como estudo piloto, o estudo transversal ICP-BR atendeu ao objetivo enunciado de avaliar a consistência e a operacionalidade da base de dados e dos sistemas de rastreamento pós-alta, avaliando 1.249 pacientes provenientes de oito hospitais em território brasileiro.' O sistema é eficiente e permitirá a avaliação da prática clínica em intervenção coronária percutânea no Brasil se alguns itens forem considerados, como descrito a seguir:

a) Inclusão de todos os hospitais brasileiros que realizam intervenção coronária percutânea. Dessa maneira teremos, com certeza, a prática clínica em intervenção coronária percutânea no Brasil. A não inclusão de todos os hospitais assim como a falta de amostragem adequada de centros que representem a prática clínica nacional tornarão as informações disponíveis insuficientes para refletir nossa realidade. As informações oriundas desse estudo piloto podem apenas representar a prática dos hospitais participantes, não devendo ser extrapolada como prática clínica em intervenção coronária percutânea no Brasil.

b) Assegurar que pacientes consecutivos sejam realmente incluídos, evitando assim potenciais vieses de seleção cujo impacto pode invalidar os resultados obtidos. Auditorias, por meio de visitas de monitorias em centros específicos, poderiam validar o processo consecutivo e a qualidade dos dados.

c) Altamente recomendável que o volume de intervenções dos hospitais e dos operadores seja coletado, como também as características dos hospitais, como acadêmico e não-acadêmico, público e privado.

d) Resultados de estudos transversais, tipo registro, devem ser considerados claros e consistentes. Para tal, análises adequadas, levando-se em consideração ajustes para as variáveis pertinentes (nunca teremos ajuste perfeito, mas assim devemos proceder), são fundamentais para a correta interpretação dos resultados e consequente recomendação para a prática clínica. Apesar da citação de que o banco de dados incluía terapêutica farmacológica adjunta, não foi descrita a utilização de terapêuticas comprovadamente eficazes

1 Instituto Dante Pazzanese de Cardiologia - São Paulo, SP, Brasil.

Correspondência: Gustavo B. F. Oliveira. Av Dr. Dante Pazzanese, 500 - Vila Mariana - São Paulo, SP, Brasil - CEP 04012-909

E-mail: oliveiragbf@gmail.com

Recebido em: 20/9/2010 - Aceito em: 21/9/2010 
com impacto confirmado em desfechos clinicamente relevantes: aspirina, clopidogrel, inibidores da enzima conversora da angiotensina, estatinas, betabloqueadores, heparinas, etc.

Finalmente, registros constituem ferramenta valiosa para documentação e otimização da prática clínica quando bem planejados, adequadamente conduzidos e corretamente interpretados. Durante os últimos 10 anos, por meio do Registro GRACE ${ }^{2-8}$, após a inclusão de aproximadamente 100 mil pacientes com síndromes coronárias agudas em 20 países, foi possível oferecermos novas evidências científicas com impacto clínico sobre a prática médica diária, por meio de 112 publicações até setembro de 2010. Algumas dessas informações relevantes incluíram o desenvolvimento e a validação de modelo estatístico para estratificação de risco em síndromes coronárias agudas, com poder discriminativo adequado para predizer mortalidade e infarto agudo do miocárdio, contemplando variáveis biológicas e perfil de pacientes com gravidade maior e mais comorbidades comparativamente aos derivados de estudos clínicos randomizados, incidência e preditores de complicações hemorrágicas, inadequação ou falta da implementação de diretrizes baseadas em evidências, tendências temporais da utilização de estratégias invasiva ou não-invasiva, terapias antitrombóticas e mortalidade, entre outros.

Por meio da implementação do conhecimento de que já somos detentores, permitiremos impacto relevante sobre doenças cardiovasculares substancialmente maior que qualquer outro novo tratamento ou intervenção. Inovar é implementar medicina baseada em evidências na prática clínica.

\section{CONFLITO DE INTERESSES}

Os autores declararam inexistência de conflito de interesses relacionado a este manuscrito.

\section{REFERÊNCIAS}

1. Paula LJC, Lemos PA, Medeiros C, Marin-Neto JA, Figueiredo $\mathrm{GL}$, Polanczyk CA, et al. Construção e validação de um Sistema Integrado de Dados de Intervenção Coronária Percutânea no Brasil (Registro ICP-BR): perfil clínico dos primeiros 1.249 pacientes incluídos. Rev Bras Cardiol Invasiva. 2010; 18(3):256-62.

2. Granger CB, Goldberg RJ, Dabbous O, Pieper KS, Eagle KA, Cannon CP, et al. Predictors of hospital mortality in the Global Registry of Acute Coronary Events. Arch Intern Med. 2003;163(19):2345-53.

3. Spencer FA, Montalescot G, Fox KAA, Goodman SG, Granger CB, Goldberg RJ, et al. Global Registry of Acute Coronary Events (GRACE) Investigators. Delay to reperfusion in patients with acute myocardial infarction presenting to acute care hospitals: an international perspective. Eur Heart J. 2010; 31(11):1328-36.

4. Oliveira GBF, Avezum A, Frederick FA, Budaj A, Dabbous $\mathrm{OH}$, Goodman SG, et al. Use of proven therapies in nonST-elevation acute coronary syndromes according to evidencebased risk stratification. GRACE Investigators. Am Heart J. 2007;153(4):493-9.

5. Fox KAA, Goodman SG, Anderson FA, Granger CB, Moscucci $M$, Flather MD, et al. From guidelines to clinical practice: the impact of hospital and geographical characteristics on temporal trends in the management of acute coronary syndromes. The Global Registry of Acute Coronary Events (GRACE). Eur Heart J. 2003;24(15):1414-24.

6. Moscucci M, Fox KAA, Cannon CP, Klein W, López-Sendón J, Montalescot G, et al. Predictors of major bleeding in acute coronary syndromes: the Global Registry of Acute Coronary Events (GRACE). Eur Heart J. 2003;24(20):1815-23.

7. Chew DP, Anderson FA, Avezum A, Eagle KA, FitzGerald G, Gore JM, et al. Six-month survival benefits associated with clinical guideline recommendations in acute coronary syndromes. GRACE Investigators. Heart. 2010;96(15):1201-6.

8. Fox KAA, Carruthers K, Steg PG, Avezum A, Granger CB, Montalescot $G$, et al. Has the frequency of bleeding changed over time for patients presenting with an acute coronary syndrome? The Global Registry of Acute Coronary Events. Eur Heart J. 2010;31(6):667-75. 